CONTRIBUCIÓN

\title{
AL ESTUDIO JURÍDICO DE LOS DERIVADOS EN EL DERECHO FRANCÉS*
}

\section{THE FRENCH LAW \\ ON FINANCIAL DERIVATIVES}

\author{
Antoine Gaudemet ${ }^{* *}$
}

\section{RESUMEN}

Pese a su creciente importancia en la economía moderna, los derivados financieros rara vez han sido objeto de un análisis jurídico completo en el Derecho Civil. Este estudio tiene la intención de remediar esta situación. Se basa en la suposición de que los derivados están siendo diseñados para transferir el riesgo que se asocia con el valor futuro de un activo separado del propio activo. A partir de este punto, los orígenes legales de los derivados, de acuerdo con la ley civil, pueden ser identificados y estos instrumentos pueden clasificarse a pesar de las numerosas formas que adoptan en la práctica. De esta forma, los derivados aparecen no solo como una comunidad de acuerdos que persiguen el mismo objetivo sino que, también, constituyen una categoría legal sobre la base de un proceso específico: cada derivado busca separar el valor de un activo subyacente del propio activo a fin de permitir a las partes cubrir o especular.

Palabras clave: Colateral, derivados, instrumentos financieros, liquidación por compensación, riesgo de sistema.

* Resumen de la tesis de Antoine Gaudemet. Les dérivés. Paris: Economica. 2010, la cual recibió los siguiente premios: Premio de tesis de la Universidad Panthéon-Assas, premio Albert Wahl de la Cancillería de las Universidades, Óscar de Derecho de Sociedades y de la Bolsa, premio de la Asociación Europea por el Derecho Bancario y Financiero.

** Profesor de la Universidad Paris II (Panthéon-Assas). Dirección postal: 12, Place du Panthéon 75005, Paris. Correo electrónico: antoine.gaudemet@u-paris2.fr. Artículo recibido el 28 de agosto de 2015 y aceptado para su publicación el 14 de diciembre de 2015. La traducción del francés estuvo a cargo de Fabricio Mantilla Espinosa, profesor de la Universidad del Rosario, Colombia. 


\begin{abstract}
Despite their increasing importance in modern economics, derivatives have seldom been subject to thorough legal analysis under civil law. This study intends to remedy this situation. It is based on the assumption that derivatives are being designed to transfer the risk one associates with the future value of an asset separately from the asset itself. From this point, the legal origins of derivatives under civil law can be identified and these instruments can be classified albeit the numerous forms they take in practice. It then appears that derivatives not only constitute a community of agreements pursuing the same aim but also make up for a legal category based on a specific process: every derivative seeks to separate the value of an underlying asset from the asset itself so as to allow the parties to hedge or speculate.
\end{abstract}

Key-words: Collateral, derivatives, financial instruments, close-out netting, systemic risk.

Las expresiones "derivados financieros", "instrumentos derivados" o, simplemente, "derivados" designan un conjunto de convenciones y títulos negociables que aparecieron en la práctica financiera a partir de la década de 1960. Su importancia económica ya no requiere demostración: normalmente se considera que los derivados tuvieron un papel fundamental en el origen y propagación de la crisis financiera actual. Su análisis, sin embargo, continúa siendo incierto.

Como se trata de una creación que proviene de la práctica, el primer reflejo es dirigirse a los operadores jurídicos mismos para comenzar la investigación. Esta búsqueda, empero, llega pronto a su fin. En efecto, para la mayor parte de ellos, los derivados son "contratos cuyo valor es

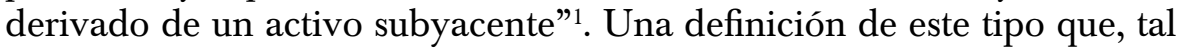
vez, puede ser suficiente para los economistas, no basta para satisfacer el análisis jurídico. Que el objeto de un contrato consista en el valor de una cosa es algo que puede concebirse fácilmente: de esta manera se ha explicado el contrato de prenda ${ }^{2} y$, en general, todas las garantías reales ${ }^{3}$. Ahora bien, lo que sí parece extraño al análisis jurídico es el hecho de

${ }^{1}$ Por ejemplo, IsAacs y Martin (1993), $V^{\circ}$ Derivative: "a financial instrument valued according to the expected price movements of an underlying asset”. Esta definición fue retomada por LEgEAIS (2009), p. 522.

${ }^{2}$ En especial, Atias (1994), p. 72. Según este autor, el objeto del contrato de prenda sería "distracción" del valor de la cosa pignorada para beneficio del acreedor prendario.

${ }^{3}$ En particular, CABRILlaC et al. (2007), pp. 369-370. 
que se defina mediante referencia a su "valor", es decir, al precio que un tercero aceptaría pagar o estaría dispuesto a recibir para sustituirse en los derechos y obligaciones de las partes en el contrato.

Resulta, entonces, evidente que los derivados no son la misma cosa para todos aquellos financieros que fungen como juristas. Para algunos solo puede tratarse de contratos, cuya terminología resulta, por cierto, inhabitual ("contratos de opción", "acuerdos de recompra", "contratos de swap" $)^{4}$, sin embargo, no parece haber consenso respecto del listado exacto de estos contratos. Para otros, también hay que incluir ciertos títulos negociables que pueden circular a través de una cesión simplificada: los "títulos derivados".

Aturdidos por las explicaciones de la práctica, se podría pensar que encontraríamos apoyo en la obra del legislador, a priori, más familiarizado con las categorías y definiciones jurídicas. Pero del aturdimiento se pasa al vértigo: el Derecho Comunitario europeo establece una lista "de instrumentos derivados" $"$, la cual fue retomada, casi al pie de la letra, por el Derecho francés, solo que su denominación fue reemplazada por "instrumentos financieros a plazo" -"instruments financiers à terme"-. Bajo este nuevo apelativo se encuentran ahora, reunidos y revueltos, en el artículo D. 211-1 A del Código Monetario y Financiero:

"1. Contratos de opción, futuros, permutas, acuerdos de tipos de interés a plazo y otros contratos a plazo relativos a instrumentos financieros, divisas, tasas de interés, rendimientos, índices financieros o medidas financieras que puedan liquidarse en especie o

4 “Note de présentation de la convention-cadre et des additifs". FBF (1994), p. 1.

También, en este sentido, el art. 1.1 de la Reglas del Mercado de Euronext: un instrumento derivado es "todo instrumento que no revista la forma de un Título que corresponda a una de las categorías siguientes: (i) contratos de opción respecto de Títulos, mercancías o productos alimenticios; también se entienden incluidos los instrumentos de la misma naturaleza que se liquiden por el pago de la diferencia en dinero; (ii) todos los demás instrumentos (distintos de los warrants y otras clases de Títulos) cuyo valor sea determinado por referencia al mercado de Títulos, mercancías o productos alimenticios o a tasas de interés o de rendimientos o a tasas de cambio o a índices o medidas, respecto de los cuales Euronext puede decidir si son susceptibles de ser negociados en un Mercado Euronext de Instrumentos Derivados, con las reservas que incluya la Reglamentación Nacional".

${ }^{5}$ Por ejemplo, el Livre blanc du comité de droit financier de l'association Paris Europlace en Bonneau y DRummond (2005), pp. 78-79.

${ }^{6}$ La sección C del anexo I de la directiva 2004/39/CE de 2004 sobre los mercados de instrumentos financieros utiliza la expresión más general "instrumentos financieros”, pero, el Reglamento (CE) 1287/2006 de 2006, que aplica esta directiva, utiliza, directamente y en múltiples ocasiones, la expresión "instrumentos derivados". 
en efectivo; 2. Contratos de opciones, futuros, permutas, acuerdos de tipos de interés a plazo y cualquier otro contrato a plazo relacionado con mercancías que deban liquidarse en efectivo o que puedan liquidarse en efectivo a petición de una de las partes por motivos distintos del incumplimiento o de otro suceso que lleve a la rescisión del contrato; 3. Contratos de opciones, futuros, permutas y otros contratos a plazo relacionados con mercancías que puedan liquidarse en especie, siempre y cuando se negocien en un mercado regulado o en un sistema multilateral de negociación; 4. Contratos de opción, futuros, permutas y cualquier otro contrato a plazo relacionado con mercancías que puedan ser liquidados mediante entrega física, no mencionados en el numeral 3) y no destinados a fines comerciales, que presenten las características de otros instrumentos financieros derivados, teniendo en cuenta, entre otras cosas, si se liquidan a través de cámaras de compensación reconocidas o son objeto de ajustes regulares de los márgenes de garantía; 5. Contratos a plazo para la transferencia del riesgo de crédito; 6. Contratos financieros por diferencias; 7. Contratos de opción, futuros, permutas, acuerdos de tipos de interés a plazo y cualquier otro contrato a plazo relacionado con variables climáticas gastos de transporte, autorizaciones de emisión o tipos de inflación u otras estadísticas económicas oficiales, que deban liquidarse en efectivo o que puedan liquidarse en efectivo a elección de una de las partes por motivos distintos del incumplimiento o de otro supuesto que lleve a la rescisión del contrato; 8. Cualquier otro contrato a plazo con activos, derechos, obligaciones, índices y medidas no mencionados en los numerales 1) a 7) que presenten las características de otros instrumentos financieros a plazo, teniendo en cuenta, entre otras cosas, si se negocian en un mercado regulado o en un sistema multilateral de negociación, se liquidan a través de cámaras de compensación reconocidas o son objeto de ajustes regulares de los márgenes de garantía”.

Recurrir al procedimiento de la enumeración -iy vaya enumeración!- refleja el rechazo a construir una definición conceptual, general y abstracta, acorde con el ideal tradicional del Derecho francés ${ }^{7}$. Además, esta enumeración, que debería, lógicamente, ser taxativa, resulta muy amplia. Los contratos enumerados pueden adoptar formas variopintas e inusitadas: "contratos de futuros", "contratos de opción”, "contratos de permuta", "acuerdos de tipos de interés a plazo". De igual manera, las

${ }^{7}$ Sobre este ideal, véase, en particular, CoRnu (1998), p. 259, especialmente, Nº 8 y ss. 
cosas que pueden ser objeto de estos contratos son muy numerosas, casi ilimitadas: pueden ser bienes, susceptibles de apropiación y cedibles, como mercancías, instrumentos financieros y divisas, pero, también, referencias abstractas, como tasas, rendimientos, índices o variables climáticas. Es, precisamente, por esta razón que ciertos autores han concluido que la lista de "instrumentos derivados", del Derecho europeo, y aquella de "instrumentos financieros a plazo", del Derecho interno francés, estarían completa o, prácticamente, desprovistas de significado ${ }^{8}$.

Pasar de esta afirmación a concluir que los derivados o los instrumentos financieros a plazo -que parecen ser sinónimos- son reacios a toda definición resulta muy fácil ${ }^{9}$. Así y todo, se debe evitar este pesimismo radical. $\mathrm{Al}$ origen de esta sentencia inapelable tal vez se halle una premisa metodológica errada: la de querer, a como dé lugar, ordenar científicamente todo aquello que en la práctica se suele designar con el nombre de "derivados" o, en la ley, con la expresión "instrumentos financieros a plazo". Ni la práctica ni la ley son prueba fidedigna en el plano del estricto del análisis jurídico.

En efecto, en la práctica, los operadores no suelen preocuparse de la lógica jurídica cuando organizan sus actividades y el legislador, presionado por el desarrollo de los derivados, legisló sin un especial cuidado por la metodología: en cada oportunidad lo hizo para evitar que el desarrollo de un instrumento nuevo se viera entorpecido por consideraciones de orden jurídico.

Así las cosas, resulta preferible comenzar por el contexto económico que determinó la aparición y desarrollo de los derivados -el conocimiento de la necesidad económica a la cual respondieron estos instrumentos permite identificarlos-y, luego, sí buscar su identidad jurídica. Esta es la metodología que se utilizará en este estudio.

Esto pone en evidencia una importante coincidencia: los instrumentos que luego fueron denominados "derivados" se fueron desarrollando a medida que se venía abajo el orden económico establecido después de la Segunda Guerra Mundial. Un autor, que estudió los contratos de swap, demostró que los primeros derivados fueron creados a partir de $1976^{10}$. Ahora bien, el Acuerdo de Kingston, que puso fin al sistema de paridad de las divisas establecido luego de la guerra por los acuerdos de Bretton

${ }^{8}$ Por ejemplo, Gauvin (2003), p. 21.

${ }^{9}$ Esto explica, también, el hecho de que varios autores hubieran preferido circunscribir su análisis a ciertas clases de derivados. En este sentido: Chabert (1989); Valette (1990); Gauvin (1999); Gauvin (2003). Distinto es el enfoque de: Medjaoui (1996); Girino (2001); Reiner (2002); Mojuyé (2005); Pailler, (2008).

${ }^{10}$ Chabert (1989), p. 7. 
Woods, data también de $1976^{11}$. Se puede presentir que hubo aquí más que una simple coincidencia, una auténtica relación de causalidad. El abandono del sistema de paridad de las divisas, seguido por las dos crisis petroleras, trajo como consecuencia la conmoción del equilibrio económico tradicional, lo cual se tradujo en el aumento de la volatibilidad de los precios de los activos económicos: los precios de las materias primas, el valor de las acciones y obligaciones, las tasas de interés sufrieron cambios con mayor rapidez y de forma más radical que en el pasado. Esta situación generó en los agentes económicos, principalmente en aquellos involucrados en el comercio internacional, una nueva necesidad de protección: ¿̇cómo garantizar que un crédito reflejado en divisas no se afecte por la evolución del cambio? ¿Cómo garantizar que las ventajas de una deuda con interés variable no se pierdan por el aumento de la tasa de interés? En pocas palabras, ¿cómo transferir los riesgos asociados al valor futuro de una cosa, sin desprenderse de la cosa misma?

Se puede objetar que esta cuestión no es del todo nueva, a pesar de que el desarrollo del comercio internacional la tornó más apremiante ${ }^{12}$. Sin embargo, si la práctica puso en marcha la imaginación para concebir los derivados, fue, probablemente, porque los instrumentos financieros tradicionales para la transferencia de riesgos no resultaban idóneos. Instrumentos no faltaban: contratos de seguros, garantías de pagos, provisiones contables y múltiples técnicas de diversificación de portafolios. Si estos instrumentos tradicionales fueron dejados de lado para dar lugar a los derivados, es, probable, porque no permitían alcanzar el objetivo que perseguían los agentes económicos: transferir el riesgo asociado al valor futuro de una cosa, sin desprenderse de la cosa misma. A partir de esta intuición, el estudio jurídico de los derivados pudo organizarse.

Los derivados son instrumentos de transferencia de riesgos. A este título son también fuente de riesgos para las partes y terceros. La parte que, por medio de un derivado, transfiere un riesgo a su contratante podría no estar en realidad expuesta a ese riesgo, por ende, especularía con el acaecimiento del evento aleatorio. De igual manera, la parte que asume el riesgo transferido a través de un derivado podría, perfectamente, no estar en las mismas condiciones que su contratante para asumirlo. En cuanto a

11 "La Conferencia de Kingston, reunida los días 7 y 8 de enero de 1976, pone fin, de forma definitiva, al sistema de Bretton Woods: el comité interino de gobernadores del Fondo Monetario Internacional (FMI) aprobó, en Jamaica, la segunda enmienda a los estatutos del FMI, a saber la total libertad de régimen de cambio para cada uno de sus miembros".TEULON (2004).

${ }^{12}$ El Chicago Board of Trade introdujo, desde 1865, la negociación de contratos de opción estandarizados para las transacciones de granos. Sobre este punto, consúltese: DidiER (1999), pp. 281-282. 
los resultados, el incumplimiento de sus obligaciones por una de las partes podría desencadenar el de otras personas en relación directa o indirecta con ella: en materia de derivados, el riesgo de contraparte se transforma en "riesgo del sistema", es decir, en riesgo para todo el sistema financiero.

Así las cosas, se puede dimensionar la amplitud y dificultad de la materia, la cuales se pueden resumir con esta idea: los derivados son instrumentos de transferencia de riesgos (primera parte) y, consecuencialmente, fuente de riesgos para las partes y terceros (segunda parte).

\section{PRIMERA PARTE:}

LOS DERIVADOS, INSTRUMENTOS DE TRANSFERENCIA DE RIESGOS

Los derivados permiten transferir los riesgos asociados al valor futuro de una cosa, sin desprenderse de la cosa misma. Empero, es bien sabido que existen otros instrumentos tradicionales para la transferencia de riesgos, como los contratos de seguros y las garantías de pago. Así, pues, la primera pregunta que, naturalmente se atañe a la identidad jurídica de los derivados: ¿tienen estos instrumentos características indentificatorias que permitan diferenciarlos de otros de transferencia de riesgos?

Pero para precisar la identidad de los derivados, es necesario identificarlos previamente: antes de abordar su análisis jurídico, hay que circunscribir el alcance de nuestro análisis. Así las cosas, después de haber identificado los derivados (título I) podremos comenzar la búsqueda de su identidad (título II).

\section{Título I: \\ la identificación de los derivados}

De acuerdo con el significado corriente, la identificación es "la acción de reconocer una cosa como tal"13. Se trataría, entonces, de reconocer cuáles son los derivados, pero la tarea no es nada fácil: los instrumentos que, en la práctica, se suelen conocer como "derivados" son muy numerosos y los diferentes listados de ellos que se han propuesto están muy lejos de la unanimidad. De igual manera, el legislador, en su enumeración de los "instrumentos financieros a plazo", no ha hecho gala de austeridad ni de precisión, como ya se ha expresado. Por tal razón, lo más conveniente, a la hora de identificar los derivados, es remontarse a las necesidades de los agentes económicos -transferir el riesgo asociado al valor futuro de una

\footnotetext{
${ }^{13}$ Guilbert (1971), $V^{\circ}$ Identification.
} 
cosa, sin desprenderse de la cosa misma- y, de forma abstracta, buscar los instituciones jurídicas capaces de satisfacerlas.

Pero, incluso, después de haber identificado las instituciones jurídicas de las cuales se sirven los derivados, seguimos frente a un gran número de instrumentos que obedecen a reglas diferentes. Esta gran variedad reclama una clasificación jurídica, es decir, la búsqueda de un orden lógico que permita la aplicación distributiva de las normas jurídicas a los derivados.

La determinación de las instituciones de las cuales se sirven los derivados (capítulo I) debe, entonces, completarse mediante una clasificación jurídica de los derivados (capítulo II).

Capítulo I:

Las instituciones de las cuales se sirven los derivados

Tres instituciones conocidas de vieja data permiten satisfacer la nueva necesidad que sintieron los agentes económicos a partir de la década de 1960: el plazo suspensivo, la promesa unilateral de contrato y el acuerdo interno de cesión de deuda o de crédito.

La estipulación de un plazo suspensivo permite a una parte determinar, desde el momento mismo de la celebración de un contrato, el valor esperado de una cosa al vencimiento de un plazo. La promesa unilateral de contrato confiere a su beneficiario el derecho de exigir al promitente la celebración de un contrato en el futuro, contrato cuyas condiciones fueron establecidas de manera previa. Finalmente, el acuerdo interno de asunción de deuda o cesión de crédito permite al deudor o al acreedor de una obligación transferir a un tercero cesionario la contribución a una deuda o la prestación de un derecho de crédito, sin realizar la transferencia del vínculo obligacional.

Así y todo, estas tres instituciones, en términos estrictos, no alcanzan a garantizar resultados del todo equivalentes. En efecto, las dos primeras solo permiten alcanzar el objetivo mediante un acondicionamiento: cuando el riesgo que se va a transferir nace de un bien, es decir, de una cosa apropiable y cedible, la transferencia de ese bien en ejecución del contrato debe excluir de forma definitiva o alternativa. De lo contrario, el bien en cuestión sería transferido junto con el riesgo que lo afecta. El acuerdo interno de asunción de deuda o cesión de crédito, por su parte, permite alcanzar directamente el objetivo perseguido: es de su esencia misma que la deuda o el crédito no se transfieran al tercero, quien solo acepta la mera contribución a la deuda o la participación en las ganancias del crédito, sin recibir el vínculo obligacional.

Tres suertes de contratos permiten, entonces, alcanzar el objetivo perseguido por los agentes económicos: los contratos a plazo, en los cuales la transferencia de la cosa subyacente se excluye, definitiva o alternativa- 
mente, por el pago de una suma de dinero; las promesas unilaterales de contrato, en las cuales la transferencia de la cosa subyacente se excluye del contrato prometido, definitiva o alternativamente; por último, los acuerdos internos de asunción de deuda o cesión de crédito. Desde la perspectiva del estricto análisis jurídico, solo estos contratos deberían constituir derivados, incluso si las clasificaciones del derecho comunitario, "instrumentos derivados", y del derecho interno, "instrumentos financieros a plazo", suelen ir más allá.

\section{Capítulo II:}

La clasificación de los derivados

El conocimiento de las instituciones jurídicas de las cuales se inspiran los derivados no basta para identificar estos instrumentos. Para complementarlo, es necesaria una clasificación que dé cuenta de la forma cómo se organizan y distribuyen estos instrumentos que, evidentemente, están regidos por reglas distintas.

En la práctica, se han elaborado múltiples propuestas de clasificación, las cuales, por regla general, no parecen haberse preocupado mucho por los presupuestos de la lógica jurídica. Normalmente, parten de la naturaleza de la cosa subyacente (ya sea que se trate o no de un bien) ${ }^{14}$, del mercado del cual provienen los derivados (mercado reglamentado o de contratación directa) o de las instituciones jurídicas en las cuales se basan los derivados ("contratos de opción", "acuerdos de recompra", "contratos de swap") ${ }^{15}$.

Así y todo, realizar una clasificación en estricto jurídica de los derivados sí parece posible. En efecto, para tal fin, se propone distinguir primero si los derechos que nacen de un derivado están o no plasmados en un título negociable; luego, diferenciar si los derechos se hallan en firme o son meramente potestativos y, finalmente, si la cosa subyacente es o no susceptible de ser transferida ${ }^{16}$. Estos tres criterios son de aplicación sucesiva: el primero, permite establecer la división principal entre "títulos

${ }^{14}$ Igualmente, la presentación que hacen algunos autores en "generaciones sucesivas" de derivados, la cual no es sustancialmente distinta: LAUwick (1994), p. 32; VAuplane y BORnet (2005), p. 629.

${ }^{15}$ Por ejemplo, la presentación de Hudson (2006), p. 28 ss. Incluso, el legislador francés fue sensible a esta presentación, puesto que, en los términos del art. D. 211-1-A del Código Monetario y Financiero, los instrumentos financieros a plazo pueden ser, alternativamente, "contratos de opción", "acuerdos de recompra" o "permutas".

${ }^{16}$ Esta clasificación retoma, en parte, aquella presentada por Thierry Bonneau y France Drummond, quienes distinguen, dentro de los instrumentos financieros, los "títulos financieros" y los "contratos financieros", luego, dentro de los contratos financieros, los "futuros" ("contratos simples et contratos de swap") y los "contratos de opción". BONNEAU y DRUMMOND (2005), pp. 78-79. 
derivados" y "contratos derivados"; luego, en el seno de estas dos categorías, el segundo criterio sirve para diferenciar dos subconjuntos que, a su turno, se dividen mediante la aplicación del tercer y último criterio. Así las cosas, los derivados pueden ser distribuidos en diferentes clases y esta clasificación permite una aplicación distributiva de las normas jurídicas: para cada estadio de la división, se aplicará o no un conjunto completo y coherente de normas, lo cual dependerá de la calificación en la subdivisión correspondiente ${ }^{17}$. Una vez clasificados los derivados es posible determinar su identidad jurídica.

\section{Título II: la identidad de los derivados}

Por "identidad" se entiende el conjunto de rasgos que confiere su individualidad a una institución jurídica: se trata, entonces, de identificar los elementos que unifican los instrumentos que han sido identificados como "derivados" -suponiendo que estos elementos existan-. Para tal efecto, podemos servirnos, una vez más, de la misma metodología: tomar como punto de partida el hecho de que el fin de los derivados es transferir el riesgo asociado al valor futuro de una cosa, sin desprenderse de la cosa misma. En este orden de ideas, los derivados constituyen, por lo menos, una "comunidad" de contratos unidos por su objeto contractual.

Pero esta primera fase del razonamiento, aunque necesaria, no es suficiente para determinar de forma completa la identidad jurídica de los derivados. Una "comunidad" de objeto contractual no constituye, necesariamente, una categoría de contratos. Contratos con el mismo objeto pueden, sin embargo, corresponder a categorías jurídicas diferentes. Se entiende por "categoría" un "conjunto distintivo de elementos que presentan características similares" ${ }^{18}$. Ahora bien, para reconocer su existencia es necesario diferenciar estos elementos de otros conjuntos jurídicos, es decir, de las calificaciones vecinas. En el caso de los derivados, la cuestión reviste especial importancia puesto que han sido objeto de las más variopintas calificaciones: desde contratos de juego y apuesta, hasta garantías de pago, pasando por contratos de seguro. Así las cosas, solo puede definirse su categoría jurídica mediante una distinción de principio entre los derivados y las demás calificaciones.

${ }^{17}$ Cornu (2004), $V^{\circ}$ Clasificación: "operación que consiste en reagrupar sistemáticamente los elementos homogéneos o heterogéneos de un grupo, dentro de un cuadro racional que incluye una división mayor basada en un criterio dominante y unas subdistinciones basadas en diversos criterios combinados, con el fin de someter al análisis, en abstracto, una referencia elaborada".

${ }^{18}$ Op. cit., $V^{\circ}$ Categorie. 
En otras palabras, la existencia de los derivados como comunidad de contratos (capítulo I) permite vislumbrar la presencia de una categoría de contratos (capítulo II).

Capítulo I:

Los derivados, comunidad de contratos

A pesar de ser numerosos y variados, los derivados convergen todos en su objeto contractual, entendido como "la operación que las partes en un contrato buscan realizar; el interés que la convención pretende satisfacer" ${ }^{\prime 19}$. En principio, los derivados tienen todos por finalidad la transferencia de los riesgos asociados al valor futuro de una cosa, sin desprenderse de la cosa misma. Ya sea que una parte quiera descargarse de un riesgo al cual está, realmente, expuesta, en estos casos se dice que aquella "se cubre", ya sea que desee, por el contrario, asumir un riesgo al cual, de otra forma, no estaría expuesta, en estos casos se dice que aquella "especula" con la esperanza de una ganancia.

Este objeto contractual particular justifica que los derivados compartan ciertas características que dan claridad sobre su identidad jurídica. En primer lugar, los derivados son siempre contratos sinalagmáticos a título oneroso, pero estas características, demasiado generales, no parecen ser de mayor utilidad. En segundo lugar, los derivados no son instrumentos de financiamiento, son siempre contratos aleatorios y de ejecución sucesiva, además de que generan, de manera principal, obligaciones dinerarias. Son, precisamente, estas características las que resultan de mayor utilidad para esclarecer su identidad jurídica.

Ahora bien, no hay que olvidar que un objeto contractual y unas características comunes no permiten, ipso facto, conformar una categoría de contratos: el contrato de seguro y las garantías de pago pueden, de manera similar, transferir el riesgo asociado a una cosa en el futuro, sin transferir la cosa gravada. El contrato de seguro, por su parte, es un contrato aleatorio y de ejecución sucesiva cuya ejecución es principalmente monetaria. Así, pues, para determinar los contornos de una categoría jurídica propia de los derivados, resulta imperativo distinguir a los derivados de esas otras calificaciones.

${ }^{19}$ Beudant (1936), p. 124. Sobre la distinción entre el objeto de la obligación y el objeto del contrato cfr. Mazeaud (1998), p. 232; TERré et al. (2005), p. 316 ss. Sin embargo, algunos autores critican la expresión "objeto del contrato", principalmente, porque, según ellos, solo la obligación puede tener un objeto: por ejemplo, Flour, Aubert y Savaux (2009), p. 199; Malaurie, Aynès y Stoffel-Munck (2009), pp. 301-302. 
Capítulo II:

Los derivados, categoría de contratos

El objeto contractual de los derivados, en efecto, ha dado lugar a comparaciones con otros contratos que permiten transferir un riesgo sin incluir la cosa gravada, como lo son el contrato de seguro y las garantías de pago. De igual manera, la preponderancia de las obligaciones dinerarias, en el caso de los derivados, ha suscitado aproximaciones entre estos y las operaciones de crédito -mediante descubierto y operaciones de crédito documentario-.

Con todo, resulta viable distinguir entre los derivados y esos otros contratos. Algunas características diferenciadoras de los derivados lo permiten. La distinción entre los derivados y el contrato de juego y apuesta no es, en realidad, la más interesante, puesto que algunos derivados corresponden, en efecto, a la calificación de apuesta. Basta con precisar que, en el caso de los derivados, las partes tiene la posibilidad de cubrirse respecto de un riesgo preexistente, lo cual no es viable para la categoría de apuesta.

Es sobre todo la distinción entre los derivados y las operaciones de seguros y de crédito la que permite esclarecer la identidad particular de los derivados. De su comparación con el seguro se evidencia el hecho de que los derivados permiten la especulación: esto significa que, en términos generales, las partes en un derivado asumen siempre una obligación propia, pero separada del perjuicio eventual que asumiría una de ellas. Esta misma característica se encuentra, incluso más evidente, en la distinción entre los derivados y los créditos documentarios: las obligaciones de las partes en un derivado son siempre jurídicamente independientes de la cosa que subyace al contrato. En pocas palabras: la característica distintiva que se perfila con mayor claridad en los derivados es la autonomía de las obligaciones de las partes respecto de la cosa subyacente. Si bien es cierto que las obligaciones se determinan mediante referencia al valor de la cosa subyacente, esto no incide en el hecho de que aquéllas sean siempre jurídicamente independientes de la suerte de esta.

Así y todo, esta característica no parece justificar, por sí sola, la existencia de una categoría jurídica autónoma. Para que exista tal categoría es necesario vincular esta característica a una técnica original que evidencia la naturaleza misma de los derivados. Ahora, esta técnica existe: los derivados reposan, literalmente, sobre la técnica de la abstracción. De acuerdo con su uso corriente, "abstracción" es la operación que consiste en "aislar mentalmente los elementos de un objeto para considerarlos por separado" 20 . Y es esto lo que hacen las partes en un derivado: aíslan el valor de una cosa subyacente, para considerarlo por separado, con el fin

${ }^{20}$ Guilbert (1971), V’ Abstraire. Del latín abstrahere: 'distraer', 'separar'. 
de cubrirse o de especular. Resulta, pues, acertado afirmar que las partes en un derivado buscan cubrirse o especular, pero que, antes de poder hacerlo $y$, precisamente, para poder hacerlo, deben servirse de la técnica que consiste en abstraer el valor de la cosa sobre la cual reposan sus inquietudes o sus esperanzas. Es por medio de esta técnica que logran, luego, cubrirse o especular ${ }^{21}$. En este orden de ideas, la técnica de la abstracción constituye el criterio de una categoría autónoma, la categoría de los derivados, la cual puede definirse como el conjunto de contratos cuyo objeto consiste en abstraer el valor de una cosa subyacente para permitirle a las partes servirse de él para fundamentar sus esperanzas o sus temores respecto del futuro ${ }^{22}$.

Pero la técnica de la abstracción no se limita a esto; conlleva también un principio cuya vocación es determinar el régimen jurídico de los derivados. El principio de la abstracción puede ser definido como aquel en virtud del cual, en un derivado, la naturaleza de la cosa subyacente debe permanecer indiferente en el plano jurídico ${ }^{23}$. De este principio se desprenden dos reglas: la primera, que podemos denominar "regla de autonomía", establece la autonomía de las obligaciones de las partes en un derivado respecto de la cosa subyacente, el hecho de que el derecho subyacente sea cedido o embargado no tiene ninguna consecuencia frente al derivado que se halla al origen de este derecho. La segunda regla, que podemos llamar "regla de circunscripción de los derechos materiales", establece la autonomía de los derivados con respecto a las normas aplicables a la cosa subyacente, el hecho de que el derecho subyacente de un derivado esté regulado por la normativa concerniente a las sociedades por acciones que cotizan en bolsa, por ejemplo, en cuanto a lo relativo a los topes máximos de acciones o derechos de voto por accionista, no puede implicar la aplicación de estas normas al derivado mismo.

${ }^{21}$ Perrot (1947), p. 16: "Cuando hablamos de la técnica (...) designamos el procedimiento técnico destinado a promover el objetivo perseguido por la voluntad, haciéndolo penetrar en el plano jurídico".

${ }^{22}$ Girino (2001), p. 15: "La indiferencia del instrumento subyacente en términos de calificación conduce, entonces, a una primera conclusión fundamental, a saber: el reconocimiento del carácter abstracto como componente esencial y calificador de los instrumentos derivados". De igual manera, Gauvin (2003), p. 193: "Para ser calificados como instrumentos financieros a plazo, los derivados de crédito deben ser abstractos. En nuestra opinión, ésta es una condición tan esencial que nos atrevemos a afirmar que podría ser el cimiento de una definición legal, no solamente de los derivados de crédito, sino de todos los instrumentos financieros a plazo".

${ }^{23}$ Este principio no tiene relación con el principio de abstracción del Derecho alemán (Abstraktionsprinzip), que, por su parte, significa que la nulidad o la resolución de un acto generador de obligaciones (Verpflichtungsgeschäft) no repercute en el acto dispositivo (Verfügungsgeschäft), el cual sigue siendo eficaz. Sobre este principio, cfr., en francés, WITZ (1992), p. 124 ss. 
Una vez precisada la identidad jurídica de los derivados y aclarado su régimen mediante las reglas de principio, es necesario abordar lo referente al riesgo que nace de ellos.

\section{SEGUNDA PARTE: \\ LOS DERIVADOS, FUENTE DE RIESGOS}

El riesgo que nace de los derivados tiene una intensidad particular. La técnica de la abstracción en la cual se fundamentan estos instrumentos da lugar al fenómeno que los economistas conocen como "el efecto de palanca"24: al disociar el valor de una cosa, las partes pueden terminar asumiendo un riesgo por este valor, sin haber adquirido la cosa en cuestión, de tal suerte que el riesgo no guarde proporción con sus activos. Quien adquiere acciones con el deseo de revenderlas a un precio más elevado especula respecto de las fluctuaciones del valor de estas acciones, pero, en principio, solo puede especular en la medida de sus activos, puesto que, previamente, debe adquirir las acciones que espera revender, después, con una plusvalía. Por el contrario, quien celebra operaciones con derivados financieros respecto de estas mismas acciones puede especular sin estar limitado por el valor de estas; en el momento de la celebración, no está obligado a adquirir las acciones en cuestión y, sin embargo, está expuesto a las variaciones del valor de estas como si las hubiese adquirido. Así, pues, quien celebra operaciones de derivados puede asumir un riesgo mucho más importante.

Este es, precisamente, el análisis que hacen los economistas de la operación:

"los riesgos individuales respecto de los derivados financieros son de la misma naturaleza que aquéllos que se asumen en el mercado al contado. La diferencia no radica en los riesgos que implican, sino en su intensidad: la existencia del importante efecto de palanca imprime una resonancia particular a estos riesgos individuales e

24 "Vinculado al endeudamiento (el efecto de palanca) caracteriza un crecimiento de la rentabilidad de capitales propios mediante el endeudamiento. Este efecto da cuenta, en realidad, de un fenómeno simple: si la rentabilidad económica de una inversión es superior a la tasa de interés de los préstamos, la sociedad va a mejorar su rentabilidad financiera. Este primer significado financiero del efecto de palanca ha sido extendido por un segundo significado: el efecto de palanca puede resultar en un mercado financiero de posibilidades por las posibilidades ofrecidas por este mercado y multiplicar el beneficio que resulta de una operación”. CAUdamine y Montier (1998), p. 433. Es el segundo significado el cual adoptamos en este trabajo. 
intensifica la probabilidad de su transformación en un riesgo de todo el sistema" ${ }^{25}$.

Esta explicación permite relevar otro elemento de los derivados: los riesgos "individuales" que aquellos implican pueden transformarse en un "riesgo del sistema", es decir, en un riesgo colectivo. Citando, una vez más, a los economistas, por riesgo del sistema se debe entender

"un riesgo de gran magnitud que resulta de disfunciones en el sistema bancario o financiero, cuando la interacción de los comportamientos individuales, en vez de realizar ajustes correctores, alteran el equilibrio general" ${ }^{\prime 2}$.

En otras palabras: se trata del riesgo de que la falla de un intermediario financiero en el cumplimiento de sus obligaciones implique la falla en cadena de otros intermediarios. Por consiguiente, el "sistema financiero" -la colectividad de los intermediarios financieros- puede resultar afectado.

Ahora bien, los derivados no son fuente de riesgo solo para partes (título I) sino, también, para los terceros relacionados con estas (título II).

Título I:

los derivados, fuente de riesgo para las partes

Tradicionalmente, en un contrato riesgoso, los intereses de la partes pueden asegurarse tanto en la etapa de formación como en la etapa de ejecución. En este orden de ideas, lo relativo a la protección del consentimiento en los derivados (capítulo I) debe preceder a su ejecución (capítulo II).

Capítulo I:

La protección del consentimiento

La protección del consentimiento reviste especial importancia en el caso de los derivados. En efecto, parecería inconcebible que una persona asumiera las obligaciones propias de un derivado sin haber mesurado el riesgo que esto implica. Esta protección puede garantizarse en dos niveles distintos.

Por un lado, y de forma general, la calidad -capacidad y poder- de ciertas personas para celebrar operaciones con derivados puede limitarse o,

${ }^{25}$ Boissieu y Aglietta (1994), p. 62.

26 "Le risque de système" Aglietta (1991), p. 63. Esta definición fue retomada por la Comisión Bancaria: "La prise en compte du risque systémique dans la surveillance des établissements de crédit”, Commission bancaire (1991), p. 181. 
incluso, excluirse cuando los derivados presentan un carácter especulativo incompatible con los intereses que aquellas deben proteger, así, por ejemplo, de las personas naturales, pero también de ciertas personas morales, como las personas de derecho público, las compañías de seguros, etcétera.

Por el otro, cuando se reconoce capacidad y poder para celebrar las operaciones con derivados, debe garantizarse la integridad del acuerdo de voluntades de las partes. A priori, podría pensarse que las figuras de los vicios del consentimiento -error, fuerza y dolo- son suficientes, sin embargo, de acuerdo con una expresión que ya se ha vuelto corriente, existen también unas "medidas curativas" para proteger el consentimiento: su sanción, a posteriori, no resulta un disuasivo suficiente para garantizar la integridad del acuerdo de voluntades de las partes en el momento de la formación del contrato. Si se tiene en cuenta la intensidad particular del riesgo que nace de los derivados, resultan indispensables las medidas "preventivas" para asegurar la información y reflexión previas de las personas que desean $-\mathrm{y}$ pueden- celebrar operaciones con derivados.

Pero la protección del consentimiento de la partes, aunque necesaria, no es suficiente para garantizar de forma completa la ejecución de los derivados. Se trata de contratos de ejecución sucesiva que, además, son aleatorios y su ejecución no se concluye nunca.

Capítulo II:

La protección de la ejecución

Para protegerse contra el riesgo de incumplimiento de los derivados, en la práctica se ha pensado, naturalmente, en la utilización de garantías de pago. Estas han sido definidas como los

“privilegios específicos otorgados a uno o varios acreedores, cuya finalidad es remediar las irregularidades en la ejecución de una obligación o precaver su incumplimiento" 27 .

Las partes en un derivado, conscientes del riesgo que este implica, siempre pueden acompañarlo con una garantía; pero no hay que olvidar que el riesgo que nace de los derivados es no solo indeterminado sino, también, alternativo.

Indeterminado porque las resultas de un derivado, en cuanto a ganancias o pérdidas, dependen de las variaciones del valor de la cosa subyacente al contrato. Alternativo, porque las resultas probables de un

${ }^{27}$ Croce (1995), p. 238. 
derivado, en cuanto a ganancias o pérdidas, varían constantemente a lo largo de la duración del contrato: un día el resultado se perfila para una de las partes, al día siguiente, para la otra. Así las cosas, las garantías que se constituyen para asegurar la ejecución de los derivados tienen que ser susceptibles de ajustarse a esta situación, afectándose de forma alternativa, ora para satisfacer a una de las partes, ora para satisfacer a la otra. En la práctica se utiliza el nombre de "colateral" 28 de las garantías reales originales, para designarlas. Estas garantías especiales se sirven en forma amplia de la técnica fiduciaria y de los mecanismos de ajuste de la base.

Pero existe otra forma de protección para las personas que desean realizar operaciones con derivados: la exigencia de que su celebración se realice con un profesional cuya actividad esté reglamentada y vigilada, lo cual aseguraría, en principio, el cumplimiento de sus obligaciones. Antes que acompañar los derivados de garantías de pago, lo más plausible es exigir que se celebración se lleve a cabo con un contratante calificado ${ }^{29}$. Este procedimiento es bien conocido en materia de seguros y de crédito: la regla que obliga a contratar el seguro con una compañía de seguros es una regla de protección para los asegurados y aquella que exige que sea un establecimiento de crédito quien recibe los fondos es una regla de protección para los depositantes. Esto se explica por el hecho de que tanto las compañías de seguros como los establecimientos de crédito son, cada uno a su manera, entidades sometidas a autorizaciones administrativas y reglamentaciones preventivas de sus operaciones.

Una solución similar sería muy recomendable en materia de derivados. Su utilidad sería doble: por un lado, si se exige que las operaciones con derivados se celebren exclusivamente con profesionales, cuya actividad sea reglamentada y controlada, aumenta la probabilidad de que se cumplan los derivados, puesto que un profesional, en principio, constituirá garantías idóneas y, si el resultado definitivo de la operación le resultara desfavorable, sería más fácil de ejecutar; por otro lado, permitiría también garantizar la eficacia de la información precontractual. En efecto, si se exige la presencia de un profesional para las operaciones con derivados, esto implicaría que este fuera el deudor de la obligación precontractual de información. Así, pues, de cierta forma, la protección de la ejecución vendría a reforzar la protección del consentimiento.

${ }^{28}$ Sousi-Roubi (2002), $V^{\circ}$ Collateral: "Las garantías, independientemente de la forma que revistan (pignoraciones, prendas de dinero, requerimientos de margen...), vinculada a una operación financiera (...)".

${ }^{29}$ Naturalmente, los dos procedimientos pueden acumularse. 


\section{Título II: \\ los derivados, fuente de riesgo para los terceros}

En los derivados, la existencia de un riesgo de contraparte elevado entre las partes conlleva, de manera indirecta, un riesgo para los terceros que están relacionados con ellas. En efecto, si se concreta el riesgo que implica un derivado este podría, perfectamente, absorber el patrimonio entero de una de las partes, el cual es prenda general de sus acreedores. Sus acreedores se encontrarían, entonces, en serias dificultades y podrían, incluso, ser arrastrados a la quiebra. Ahora, si este fuera el único riesgo, no habría mucho de qué preocuparse: esta misma situación se presenta con todos los contratos de ejecución sucesiva; además, existen diferentes mecanismos que permiten a los acreedores salvaguardar sus intereses, como la acción oblicua, la acción pauliana y la acción directa, en los casos en que es procedente. Adicionalmente, la normativa aplicable a los procesos concursales consagra mecanismos de alerta y de salvaguarda que deben, en principio, prevenir el "efecto dominó" que podría generar la cesación de pagos de un deudor respecto de sus acreedores.

Pero, justo, el riesgo va más allá. En el caso de los derivados, el riesgo de contraparte puede ser tal que arrastre consigo no solo a los acreedores de las partes sino, además, a numerosas personas que están relacionadas, directa o indirectamente, con ellas. El riesgo que afecta a los acreedores se transforma en un "riesgo del sistema", es decir, un riesgo que afecta a todo sistema financiero.

En la práctica, se ha encontrado la forma de protegerse frente a este riesgo mediante la figura de la compensación, cuyo funcionamiento como garantía es conocido de vieja data, pero, este expediente no alcanza a ser suficiente para asegurar el riesgo del sistema. Así, pues, el mecanismo de protección concebido en la práctica (capítulo i) debe ser reafirmado por la ley (capítulo II) para conseguir la eficacia.

Capítulo I:

La protección concebida por la práctica

En la práctica, el riesgo que implican los derivados para las partes y para el sistema financiero en su conjunto llevó a que se concibiera un dispositivo de protección basado en la compensación. Esta, como bien se sabe, permite extinguir dos obligaciones recíprocas, hasta la concurrencia de sus valores: entre las partes que han celebrado una operación con múltiples derivados, el riesgo de contraparte ya no es, por consiguiente, igual al valor bruto de sus obligaciones, sino equivalente al monto neto del saldo potencial de compensación de sus deudas. En caso de incumplimiento de 
una de ellas, la compensación actuará como "talanquera" para proteger a los terceros del contagio.

El dispositivo concebido en la práctica opera en dos niveles sucesivos:

En primer lugar, el mecanismo convencional de la resciliación-compensación permite a las partes que han celebrado múltiples operaciones con derivados circunscribir el riesgo de contraparte entre ellas al saldo neto de compensación y ya no al monto bruto de sus obligaciones. Las operaciones con derivados se celebran en desarrollo de un contrato-marco que consagra un mecanismo original que combina el derecho de resciliación unilateral y la compensación. En caso de incumplimiento de una de las partes, la resciliación puede operar para el conjunto contratos celebrados entre ellas. El perjuicio que implicaría, para cada parte, la resciliación de cada uno de los contratos se evalúa de acuerdo con las condiciones del mercado. Las indemnizaciones por la resciliación se compensan en un saldo único exigible solo entre las partes.

Este mecanismo, si bien permite circunscribir el riesgo de contraparte al monto del saldo neto de compensación entre las obligaciones de las partes, no lo elimina por completo. Por tal razón, las partes pueden, en segundo lugar, celebrar, además del contrato-marco que consagra la compensación, un acuerdo en virtud del cual se obligan a gravar, periódicamente, bienes en garantía del saldo potencial de la compensación. Esta es la llamada técnica del "colateral" esta vez estructurada no para uno sino para múltiples derivados.

Sin embargo, la normativa que regula los mecanismos de ejecución, los procedimientos de insolvencia y las garantías reales parece impedir la plena eficacia de este dispositivo convencional de protección. Especialmente, las reglas propias a los procesos concursales paralizan las cláusulas de resciliación y la compensación. Por esta razón, el legislador tuvo que intervenir con el fin de superar estos escollos.

Capítulo II:

La protección reafirmada por la ley

En Francia, como en la mayor parte de los países miembros de la OCDE, el legislador ha aceptado levantar los obstáculos legales a la eficacia del mecanismo convencional de resciliación-compensación y de los acuerdos de colateral: las reglas propias a los mecanismos de ejecución, los procedimientos de insolvencia y las garantías reales han sido excluidas cada vez que estas resultaban inconvenientes. Esto justifica la existencia de un derecho especial para los derivados que deroga las normas generales ${ }^{30}$.

${ }^{30}$ Por ejemplo, LuCAS de Leyssac (1998), p. 1567. 
Los acreedores de derivados o de acuerdos de colateral gozan, por consiguiente, de un régimen favorable frente a los demás acreedores.

Derogaciones de esta importancia solo podían consagrarse a condición de emprender una lucha contra el riesgo del sistema, como un imperativo de orden público. Este esfuerzo rara vez ha sido realizado por los juristas y, a la postre, se ha dejado a cargo del análisis económico. Así y todo, hay un argumento fundamental que justifica el hecho de que pueda considerarse la lucha contra el riesgo del sistema como parte del orden público económico: el sistema financiero tiene el monopolio jurídico de la distribución del crédito $^{31}$. Desde esta perspectiva, el riesgo de que la imposibilidad de hacer frente a sus compromisos por parte de un intermediario financiero repercuta en cadena a los demás intermediarios del sistema no parece ser una situación tolerable, no habría, entonces, sustituto para distribuir el crédito en la economía, tal como lo ha demostrado la realidad reciente.

Si bien la lucha contra el riesgo del sistema, como componente del orden público económico, parece justificada, esta tiene, sin embargo, un límite necesario: las derogaciones a las normas generales solo pueden incluirse cuando exista un verdadero riesgo de crisis para el sistema financiero. Ahora bien, desde este punto de vista, el dispositivo actual de prevención del riesgo del sistema, probablemente, no está exento de críticas. Hoy día, el derecho de los derivados financieros no solo está orientado a la prevención del riesgo del sistema, sino que se ha terminado convirtiendo en una competición entre los diferentes derechos nacionales: de cierta manera, su ratio legis basculó de la prevención del riesgo del sistema a la competitividad internacional por la primacía financiera.

\section{Bibliografía CITADA}

Aglietta, Michel (1991). "Le risque de système". Revue d'économie financière. $\mathrm{N}^{\circ}$ 18, Paris, mars.

Atias, Christian (1994). "La valeur engagée (sur l'objet du gage)". RJ com. Paris, novembre.

Beudant, Charles (1936). Cours de droit civil français. Paris: Publié par R. Beudant et la collaboration P. Lerebours-Pigeonnière avec G. Lagarde, Arthur Rousseau. T. viII.

Boissieu, Christian et Michel Aglietta, (1994). "Les marchés dérivés de gré à gré et le risque systémique". Bull. COB. N ${ }^{\circ} 283$. Paris.

Bonneau, Thierry y France Drummond (2005). Droit des marchés financiers, $\mathrm{N}^{\circ} 82$, Paris: Editorial Economica.

${ }^{31}$ En particular, el art. L. 511-5, al. 1, C. 
Cabrillac, Michel, Christian Mouly, Severine Cabrillac et Philippe Petel (2007). Droit des sûretés, Paris: Editorial Lexis Nexis.

Caudamine, Guy et Jean Montier (1998). Banque et marchés financiers, Paris: Editorial Economica.

Chabert, Pierre-Yves (1989), Les swaps, tesis, Clermont-Ferrand: Editorial Clermont-Ferrand I.

Cornu, Gérard (1998). L'art du droit en quête de sagesse. Paris: Editorial Presses Universitaires de France.

Cornu, Gérard (2004). Vocabulaire juridique. Paris: Editorial Presses Universitaires de France.

Croco, Pierre (1995). Propriété et garantie, Paris: Editorial LGDJ, Bibliothèque de droit privé, T. 248.

Didier, Paul (1999). Droit commercial, La monnaie, les valeurs mobilières, les effets de commerce. Paris: Editorial Presses Universitaires de France. Thémis Droit privé, T. III.

Federation Bancaire Francaise (1994). Note de présentation de la convention-cadre et des additifs. Paris: FBF.

Flour, Jacques, Jean-Luc Aubert y Éric Savaux (2009). Les obligations. L'acte juridique. Paris: Editorial Sirey, tome I.

Gauvin, Alain (1999). Nature et régime juridique des dérivés de crédit. Thèse. Paris: Editorial Paris I.

Gauvin, Alain (2003). Droit des dérivés de crédit. Paris: Editorial Rev. Banque.

Girino, Emilio (2001). I contratti derivati. Milan: Editorial Il diritto privato oggi.

Guilbert, Louis, René Lagane et Georges Niobey (1971). Grand Larousse de la Langue française. Paris: Editorial Larousse.

Hudson, Alastair (2006). The law on financial derivatives. London: Editorial Sweet \& Maxwell.

IsAacs, Alan \& Elizabeth MARTIN (1993). Oxford Dictionary for the Business World. Oxford: Editorial University Press.

LaUwick, Vicent (1994). "De nouvelles solutions pour la gestion des risques". Revue Banque, $\mathrm{N}^{\circ}$ 548. Paris, mai.

Legeais, Dominique (2009). Sûretés et garanties du crédit. Paris: Editorial LGDJ, coll. Manuel.

LuCAS DE Leyssac, Claude (1998). "Existe-t-il un droit spécial des produits dérivés?”. Suppl. $\mathrm{N}^{\circ}$ 133. Paris: Editorial D. Affaires.

Malaurie, Philippe, Laurent Aynes et Philippe Stoffel-Munck (2009). Les obligations. Paris: Editorial LGDJ.

Mazeaud, Lèon, Henri et Jean (1998). Obligations, théorie générale. Paris: Editorial Montchrestien, T. II, vol. 1.

Medjaoui, Khadija (1996). Les marchés à terme dérivés et organisés d'instruments financiers, étude juridique. Paris: Editorial Bibliothèque de Droit Privé. 
Mojuye, Joseph-Benjamin (2005). Le droit des produits dérivés financiers en France et aux États-Unis. Paris: Editorial Bibliothèque de Droit Privé.

PAILler, Pauline (2008). La notion d'instrument financier à terme. Thèse. Paris: Editorial Paris I.

Perrot, Roger (1947). De l'influence de la technique sur le but des institutions juridiques. Thèse. Paris: Editorial Sirey.

ReINer, Günter (2002). Derivative Finanzinstrumente im Recht. Konstanz: Editorial Nomos Verlagsgesellschaft, Baden-Baden.

Sousi-Roubi, Blanche (2002). Lexique de la banque et des marchés financiers. Paris: Editorial Dalloz.

Terre, François, Philippe Simler et Yves Lequette (2005). Les obligations. Paris: Editorial Dalloz.

Teulon, Frédéric (2004). Dictionnaire d'histoire, économie, finance, géographie. Paris: Editorial Major.

VALETTE, Diddier (1990). Les marchés d'options négociables (aspects juridiques). Thèse. Clermont-Ferrand: Editorial Clermont-Ferrand I.

Vauplane, Hubert et Jean- Pierre Bornet (1989). Droit des marchés financiers. Paris: Editorial Litec.

Witz, Claude (1992). Droit privé allemand, Actes juridiques, droits subjectifs. Paris: Editorial Litec, vol. 1.

Normas citadas

Código Monetario y Financiero Francés.

Directiva 2004/39/CE de 21 de abril de 2004, JOUE N ${ }^{\circ}$ L 145, de 2004.

Reglamento (CE) 1287/2006 de 10 de agosto de 2006. JOUE, N L 241, de 2006. 